\title{
Fluid-Thermal-Structural Coupled Analysis of a Radial Inflow Micro Gas Turbine Using Computational Fluid Dynamics and Computational Solid Mechanics
}

\author{
Yonghui Xie, ${ }^{1}$ Kun Lu, ${ }^{1}$ Le Liu, ${ }^{1}$ and Gongnan Xie ${ }^{2}$ \\ ${ }^{1}$ School of Energy and Power Engineering, Xian Jiaotong University, Xi'an, Shaanxi 710049, China \\ ${ }^{2}$ School of Mechanical Engineering, Northwestern Polytechnical University, P.O. Box 552, Xian, Shaanxi 710072, China \\ Correspondence should be addressed to Yonghui Xie; yhxie@mail.xjtu.edu.cn
}

Received 20 November 2013; Revised 23 January 2014; Accepted 1 February 2014; Published 23 April 2014

Academic Editor: Yonghong Wu

Copyright (c) 2014 Yonghui Xie et al. This is an open access article distributed under the Creative Commons Attribution License, which permits unrestricted use, distribution, and reproduction in any medium, provided the original work is properly cited.

\begin{abstract}
A three-dimensional fluid-thermal-structural coupled analysis for a radial inflow micro gas turbine is conducted. First, a fluidthermal coupled analysis of the flow and temperature fields of the nozzle passage and the blade passage is performed by using computational fluid dynamics (CFD). The flow and heat transfer characteristics of different sections are analyzed in detail. The thermal load and the aerodynamic load are then obtained from the temperature field and the pressure distribution. The stress distributions of the blade are finally studied by using computational solid mechanics (CSM) considering three cases of loads: thermal load, aerodynamics load combined with centrifugal load, and all the three types of loads. The detailed parameters of the flow, temperature, and the stress are obtained and analyzed. The numerical results obtained provide a useful knowledge base for further exploration of radial gas turbine design.
\end{abstract}

\section{Introduction}

Micro gas turbines are energy generators whose capacity ranges from $15 \mathrm{~kW}$ to $300 \mathrm{~kW}$. In recent years, micro gas turbines have been widely studied and used because of their typical advantages such as variable speed, high speed operation, compact size, simple operability, easy installation, and low maintenance. In 2003, Johnston et al. [1] built and tested a microscale, high-speed compressor impeller (12 mm diameter; 800,000 rpm) for feasibility in regard to aerodynamic performance. The results from a CFD code agreed with measured data very well, suggesting that that design method combined with CFD techniques could be used to develop rotors for a microscale, gas turbine engine. In the work by Ribaud [2], a thermodynamic model was developed and applied to study heat transfer characteristic in an ultra-micro turbine at different situations. McDonell et al. [3] studied the performance of a micro gas turbine at various conditions, and optimum operating parameters were obtained. Further, Rodgers [4] investigated flow characteristics and aerodynamic performance of a micro gas turbine. It was reported that the main factors that affected the gas turbine performance were speed ratio, rotor blade tip clearance, and outlet flow angle. Onishi et al. [5] used a full three-dimensional Navier-Stokes solver to design and study aerothermodynamics of a micro gas turbine. Losses due to the heat transfer to walls and skin friction were estimated, and the effects of turbine exhaust geometry and the number of blades on turbine performance were also studied. Fu et al. [6] performed numerical investigations on the aerothermodynamic design, geometrical design, and overall performance prediction of a millimeter-scale radial turbine.

In the design of micro gas turbines, the heat-flow coupling method is widely used to obtain the temperature distribution of the gas turbine blades. In the investigation by Bohn and Kusterer [7], a cooling configuration with cooling fluid ejection through two rows of holes at leading edge was numerically studied by applying a heat-flow coupling method. Bohn and Heuer $[8,9]$ presented a conjugated aerodynamic and thermal numerical investigation of a convection-cooled, 
high-pressure turbine nozzle guide vane. A heat-flow coupling simulation was conducted for the C3X and MarkII blade. Other applications of heat-flow coupling method for the investigation of the heat transfer in micro gas turbine can also be found in the works of Bohn et al. [10, 11]. Hill et al. [12] presented a model which takes into account the formation of oscillation notches on a steel surface. The authors presented a model to couple the flux flow with the heat transfer. Besides, equations correlating the temperature field in the steel and flux with the geometry of the lubricating layer are derived. Further, Wu et al. [13] developed a single domain enthalpy control volume method to solve coupled fluid flow and heat transfer with solidification problem arising from the continuous casting process. They found that their numerical method was robust in capturing the rapid change of temperature in the solidification region. The rapid change of fluid velocity near the solid-fluid interface could also be obtained.

The stress analysis for a micro gas turbine is usually necessary for safety and reliability. The load on a radial turbine consists of thermal load resulting from the temperature gradient, centrifugal load caused by the turbine rotation, and aerodynamic load. Thus, in stress analysis of thermal stress, centrifugal stress and the stress caused by the aerodynamic force should be analyzed. Ho and Paull [14] described a method to implement aerodynamic heating models into a finite element code for thermal-structural and thermal-structural-vibrational analyses of a hypersonic engine. A combined effect of varying dynamic pressure and thermal loads was considered, and thermal-structuralvibrational response of an engine was studied. Shen et al. [15] conducted a fluid-thermal-structure coupled analysis and an optimization of a turbine mortise/disc. In their work, a complete multidisciplinary method containing fluid-thermalstructure of the mortise/disc was formed, taking influence of the fluid flow and heat transfer into account. Recently, Krishnakanth et al. [16] carried out finite element analysis for the structural and thermal analysis of gas turbine rotor blades. They found that the temperature had a notable effect on the overall stresses in the turbine blades. Other relevant works involving multidisciplinary analysis and optimization design of gas turbines can be found in [17-21].

In this paper, the ANSYS 11.0 software is used for a fluid-thermal-structural coupling analysis of the micro gas turbine. At first, a heat-flow coupling analysis is conducted to obtain temperature distribution of a gas turbine blade and the aerodynamic force on the turbine blade. After obtaining the temperature distribution of the micro gas turbine, a stress analysis is then conducted considering three cases of loads: thermal load, aerodynamics load combined with centrifugal load, and all the three types of loads. Unlike the previous studies, this paper systematically studied the three cases to ensure the safety and reliability of the gas turbines.

\section{Heat-Flow Coupling Method}

2.1. Numerical Method. Usually the heat-flow coupling method is used to obtain temperature distribution of gas

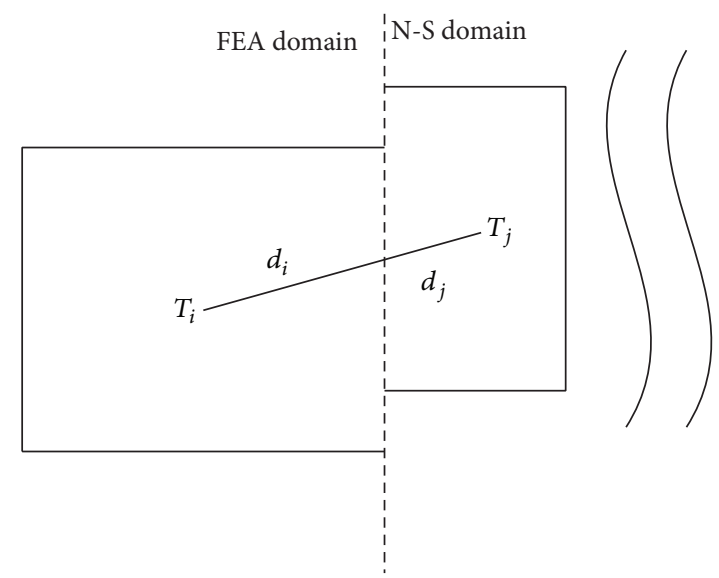

FIGURE 1: The data exchange of the coupling method at the interface.

turbine blades. This method is much better than the decoupling simulation method because it can obtain more accurate temperature field by solving the heat convection equation in the fluid domain and the heat conduction equation in the solid domain simultaneously. In this method, a NavierStokes (NS) solver for the fluid flow and a finite element analysis (FEA) for the heat conduction in the solid are used. Figure 1 shows the data exchange of the coupling method at an interface. For the fluid-thermal-structural coupled analysis of the gas turbine, the imposed aerodynamic force and temperature distribution on the turbine are obtained from the heat-flow coupling analysis. In the fluid-thermal-structural coupled analysis, the FEA mesh used for stress analysis is the same as the mesh used for the heat-flow coupling analysis. As a result, the aerodynamic force and temperature distribution on the turbine obtained from the heat-flow coupling analysis can be directly imposed on the mesh that is used for fluidthermal-structural coupled analysis.

The convective heat transfer equation is used at the interface for the data exchange of the coupling method, and it can be expressed as follows:

$$
q_{w}=h \cdot\left(T_{w}-T_{f}\right)
$$

where $q_{w}$ is the heat flux at the interface obtained by the $\mathrm{N}-\mathrm{S}$ solver, $h$ is the convective heat transfer coefficient, $T_{w}$ is the wall temperature obtained by the FEA solver, and $T_{f}$ is the flow temperature obtained by the N-S solver. The interpolation method used for the data exchange of the coupling method is expressed as follows:

$$
\begin{gathered}
T_{w}=\frac{T_{i} d_{j}+T_{j} d_{i}}{d_{i}+d_{j}}, \\
q_{w}=-k_{s} \frac{d T}{d n}=k_{s} \frac{T_{i}-T_{w}}{d_{i}},
\end{gathered}
$$

where $T_{i}$ is the temperature at the center of the element mesh in the solid domain, $T_{j}$ is the temperature at the center of the element mesh in the fluid domain, $d_{i}$ is the length of the centerline of the two elements in the solid domain, and $d_{j}$ is 
the length of the centerline of the two elements in the fluid domain.

The commercially available CFD package CFX is used for the heat-flow coupling analysis of the micro gas turbine. In the analysis of aerodynamic performance and heat transfer characteristics, the numerical interpolation method is used for the energy coupling at the interface between the flow and solid domains. To obtain the stress distribution of the turbine, a thermostructural analysis is carried out with the aerodynamic parameters and heat transfer parameters applied using the finite element package ANSYS. The nonadiabatic flow is calculated by solving the 3D steady Navier-Stokes equations, which are expressed in detail as follows.

Continuity equation is given as follows:

$$
\nabla \cdot(\rho \mathbf{u})=0
$$

Momentum conservation equation is given as follows:

$$
\nabla \cdot(\rho \mathbf{u u})=-\nabla p+\nabla \cdot \tau \text {. }
$$

Energy conservation equation is given as follows:

$$
\nabla \cdot\left(\rho \mathbf{u}\left(e+\frac{p}{\rho}\right)\right)=\nabla \cdot(\tau \mathbf{u})-\nabla \cdot \mathbf{q},
$$

where $\tau, e$, and $q$ are defined as follows:

$$
\begin{gathered}
\tau=-\frac{2}{3} \mu(\nabla \cdot \mathbf{u}) \mathbf{I}+\frac{1}{2} \mu\left(\nabla \mathbf{u}+(\nabla \mathbf{u})^{T}\right), \\
e=\widehat{u}+\frac{1}{2}(\mathbf{u} \cdot \mathbf{u}) \\
q=-k \nabla T .
\end{gathered}
$$

Hydrostatic equation in the solid domain is given as follows:

$$
K a=P_{f}+P_{S}+P_{\varepsilon_{0}}
$$

where $P_{f}$ is the volume force, $P_{S}$ is the surface force, and $P_{\varepsilon_{0}}$ is the thermal stress caused by the variation in temperature. In this paper, the thermal load $P_{\varepsilon_{0}}$ caused by the initial strain $\varepsilon_{0}$ is also considered, which is usually ignored in the previous studies.

2.2. Geometry Model of the Turbine. The geometry of the micro radial inflow gas turbine is shown in Figure 2, and the flow domain is shown in Figure 3. Table 1 shows the structural parameters of the stator cascade, and the rotor blade passage is shown in Figure 4.

2.3. Mesh Generation. The commercially available ANSYS ICEM is used for the structured mesh generation. Figure 5 shows the mesh for a single stator blade. An O-topology mesh is applied to model the blade, and the mesh around the blade is refined. For a single stator blade, there are totally $6.9 \times 10^{4}$

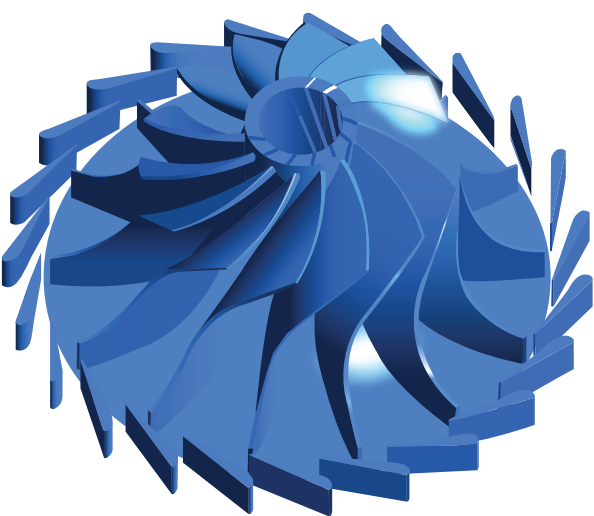

FIgURE 2: Geometry of the micro gas turbine.

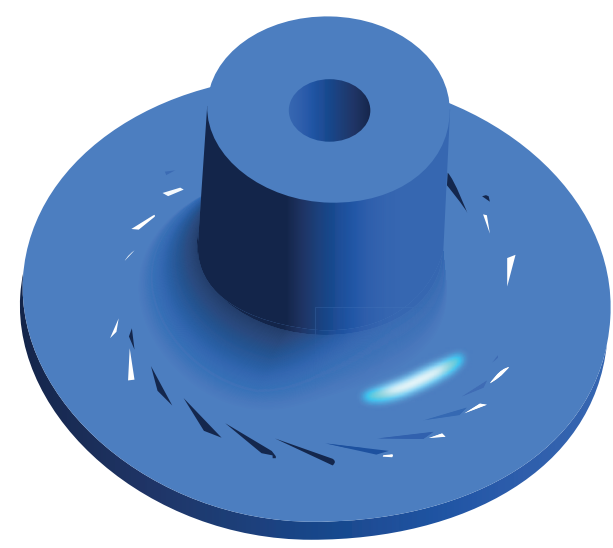

FIgURE 3: Fluid domain of the micro gas turbine.

TABLE 1: The structural parameters of the stator cascade.

\begin{tabular}{lcc}
\hline Structural parameters & Unit & Value \\
\hline Blade height & $\mathrm{mm}$ & 12.6 \\
Blade cascade & $\mathrm{mm}$ & 24.79 \\
Blade chord & $\mathrm{mm}$ & 32.9 \\
Axial chord length & $\mathrm{mm}$ & 15.5 \\
Flow inlet angle & ${ }^{\circ}$ & 35 \\
Average diameter & $\mathrm{mm}$ & 196.23 \\
Number of blades of the whole cycle & & 23 \\
\hline
\end{tabular}

and $2.0 \times 10^{4}$ cells in the fluid domain and the solid domain, respectively.

Figure 6 shows the mesh for a single rotor blade. A refined O-topology mesh is also applied around the rotor blade. Considering the tip clearance effects on the flow, the mesh in the tip clearance is also refined. Finally, there are $1.84 \times 10^{5}$ nodes in the flow domain for a single rotor blade. The mesh for the rotor blade in the solid domain is shown in Figure 7. As observed, the mesh at the blade root and the leading and trailing edge of the blade is refined for accurate simulation results. There are $4.1 \times 10^{4}$ nodes in the solid domain for a single rotor blade. Totally, there are $5.19 \times 10^{6}$ nodes in the computational domain. 


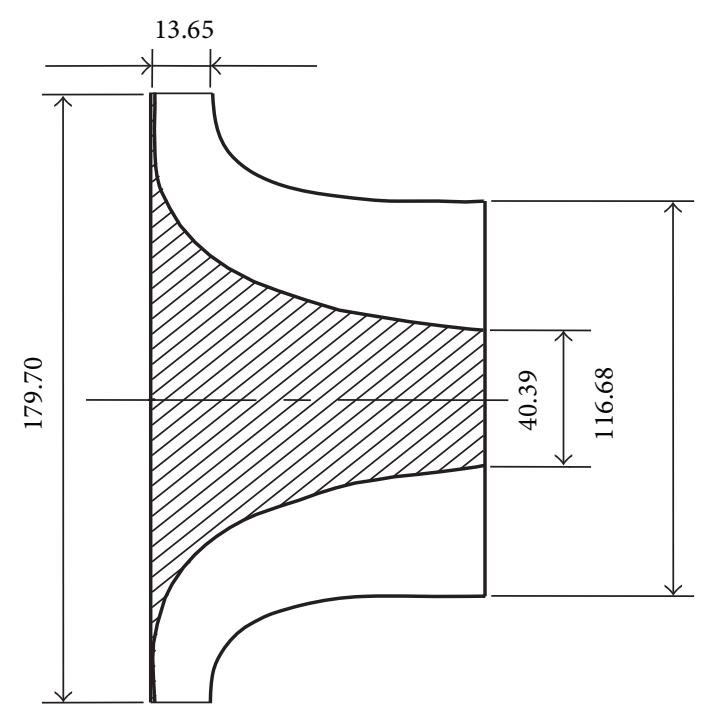

FIGURE 4: The rotor blade passage of the micro gas turbine.

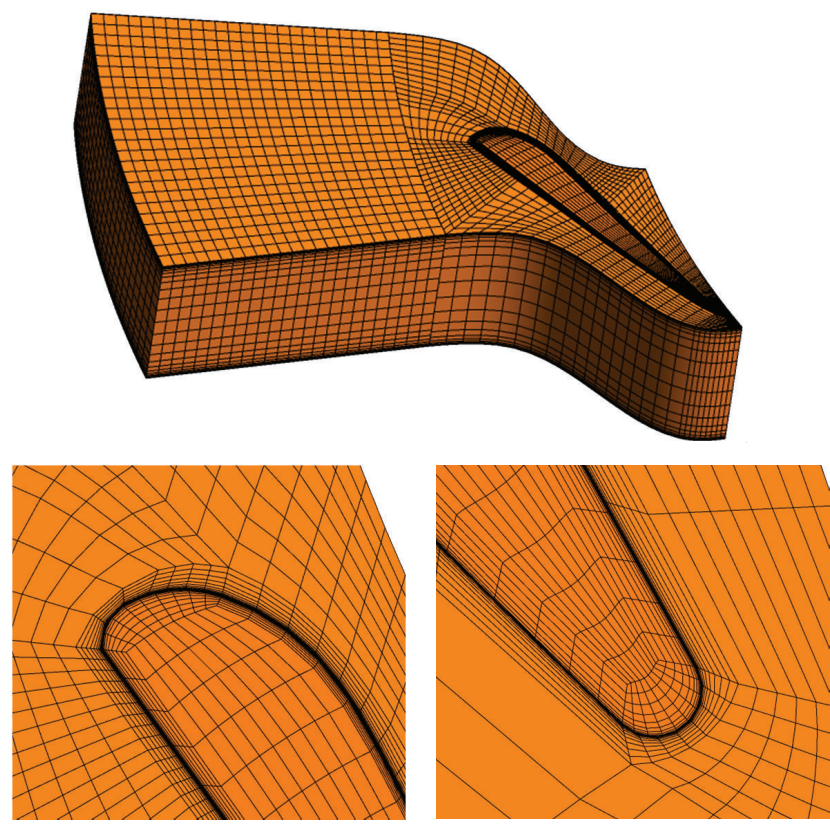

FIGURE 5: Mesh for a single stator blade.

2.4. Boundary Conditions and Validation Study. In the heatflow coupling analysis of the micro gas turbine, four computational domains are used: flow domain of the stator blade, solid domain of the stator blade, flow domain of the rotor blade, and solid domain of the rotor blade. The inlet total temperature and pressure are $930^{\circ} \mathrm{C}$ and $342037 \mathrm{~Pa}$, respectively, and the outlet pressure is $109920 \mathrm{~Pa}$. Computations were carried out at a design condition of $800,000 \mathrm{rpm}$ and at the test condition of $60000 \mathrm{rpm}$.

In the simulation, the general grid interface (GGI) connection is used for the interface between the flow domain and the solid domain, and the general connection method is used for the interface between the outlet of the stator
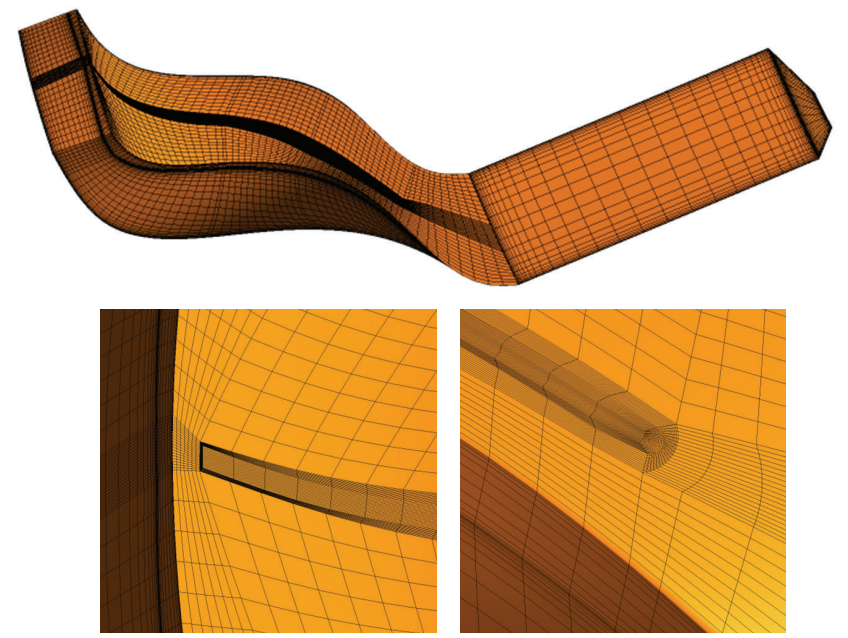

FIGURE 6: Mesh for a single rotor blade.

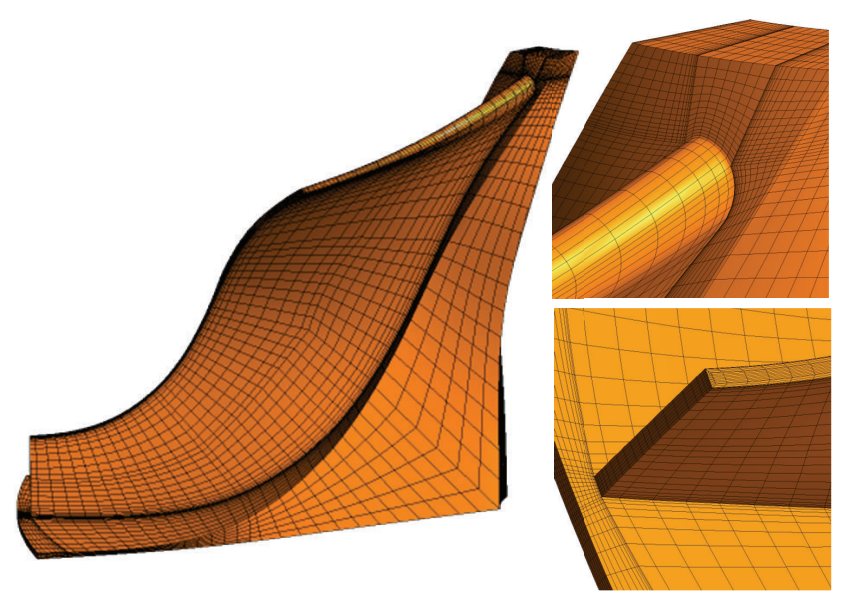

FIgURE 7: Mesh for the rotor blade in the solid domain.

blade flow domain and the inlet of the rotor blade flow domain. The solid boundaries are assumed to be adiabatic, and a no-slip boundary condition is applied along the solid boundaries. Only conduction and convection are taken into account. Radiation is neglected since differences in surface temperatures are small. The SST turbulence model with $\gamma-\operatorname{Re}_{\theta}$ transition model developed by Menter [22] is used in all the computations.

The heat-flow coupling method used in this paper has been validated by simulating the experimental study of surface heat transfer distributions of a turbine vane with film cooling by Hylton et al. $[23,24]$. The simulation is conducted for the C3X turbine vane at the experimental condition of Test 5422. Figure 8 shows the dimensionless pressure distribution along the vane surface. As observed, our computational result agrees with the experimental results by Hylton et al. [23, 24] very well.

2.5. Results and Discussions. In this section, the flow field and temperature field in the blade passage are analyzed by heatflow coupling method. The flow pattern and heat transfer 


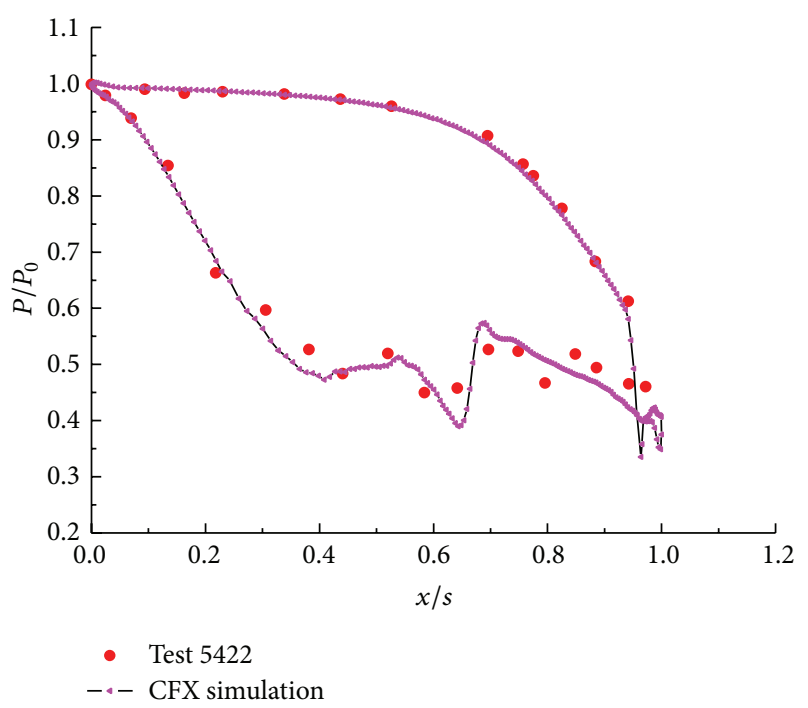

FIGURE 8: Dimensionless pressure distribution along the vane surface.

characteristics are investigated. Figure 9 shows the velocity distribution and streamline in the middle section of the stator blade. It can be observed that the flow velocity at the inlet is quite uniform, and a notable change in flow velocity distribution occurs in the blade passage. After entering the blade passage, the flow direction changes and a stagnation zone can be observed at the pressure surface around the leading edge. The stagnation point is located at $10 \%$ chord from the leading edge. The flow area decreases along the blade passage, leading to an increase in the flow velocity. A maximum flow velocity of $500 \mathrm{~m} / \mathrm{s}$ is observed at $40 \%$ chord from the leading edge. The flow velocity at the outlet of the stator blade is relatively high and uniform.

Figure 10 shows the pressure distribution in the middle section of the stator blade. A significant increase in pressure is observed at the pressure surface around the leading edge. The pressure gradient at the suction surface around the leading edge is relatively high, and the pressure decreases from about $320000 \mathrm{~Pa}$ to $220000 \mathrm{~Pa}$. The minimum pressure is observed at $40 \%$ chord from the leading edge.

The blade profile of the turbine varies along the blade height. Three typical sections along the blade height are chosen for a better investigation of the flow pattern and heat transfer characteristics. To study the flow pattern around the blade, the velocity distribution and local streamline for the sections of $10 \%, 50 \%$, and $90 \%$ blade height are shown in Figure 11. In the section of $10 \%$ blade height, flow separation around the blade is observed, and the stagnation point is located around the leading edge of the pressure surface, where a vortex can be observed. The decrease in pressure and increase in flow velocity result in a maximum velocity of $350 \mathrm{~m} / \mathrm{s}$ around the trailing edge, where notable velocity gradient can be observed. At the suction side, the flow is q steady, and the variation in the flow velocity is relatively smooth. No flow separation can be found at the suction side except for the counter-rotating vortex pair at the trailing edge. In the section of $50 \%$ blade height, a higher maximum flow velocity of $420 \mathrm{~m} / \mathrm{s}$ is observed, and there is no vortex at the leading and trailing edge of the blade. In the section of $90 \%$ blade height, the tip clearance has notable effects on the flow, leading to the inhomogeneous flow around the blade.

Figure 12 shows the pressure distribution for the section of $10 \%, 50 \%$, and $90 \%$ blade height. A relatively high pressure gradient is observed at the inlet. In the section of $10 \%$ and $50 \%$ blade height and in the section of $90 \%$ blade height, the distribution of the isopiestic line is quite different. At the leading edge, a significant pressure gradient occurs, and the flow state changes dramatically. This can be attributed to the tip leakage flow caused by the tip casing scraping flow and pressure difference in the tip clearance [25].

The dimensionless pressure and temperature are used to analyze the flow field. The dimensionless pressure and temperature are the ratios of the pressure and temperature of the rotor blade surface to the inlet pressure and temperature of the rotor stage, respectively. Figure 13 shows the dimensionless pressure distribution on the turbine blade. A maximum dimensionless pressure of 0.62 can be observed at the leading edge of the pressure surface. The surface pressure decreases in the flow direction. The dimensionless pressure at the pressure surface is basically higher than 0.42 , and the dimensionless pressure at the suction surface is relatively lower with a maximum value of 0.4 at the inlet. Besides, the pressure gradient at the pressure surface is relatively high, and the minimum dimensionless pressure occurs at the trailing edge. At the trailing edge of the suction side, a notable pressure gradient in the radial direction is observed, resulting from the increase in blade height and blade twist.

The analysis of blade temperature is very important because it has a notable effect on the thermal stress calculation and blade life prediction. Figure 14 shows the dimensionless temperature distribution on the turbine blade. As observed, the dimensionless temperature at the inlet is relatively high because of the convection heat transfer between the blade and hot gas. In the blade passage, the gas does work by expansion, leading to a decrease in the pressure and temperature along the blade passage. A maximum dimensionless temperature of 0.89 is observed at the inlet, and the minimum dimensionless temperature occurs at the blade root around the trailing edge.

\section{Coupled Fluid-Thermal-Structural Analysis}

3.1. Boundary Conditions and Solution Methods. The commercially available FEM package ANSYS 11.0 is used for the stress analysis of the rotor blade, based on the heatflow coupling analysis above. The FEM mesh used for stress analysis is obtained from the results of the heat-flow coupling analysis; thus, it is the same as the mesh used for heat-flow coupling analysis, as shown in Figure 15.

The load on the radial turbine in this paper consists of thermal load resulting from the temperature gradient in the turbine, centrifugal load caused by the centrifugal force, and aerodynamic load. 

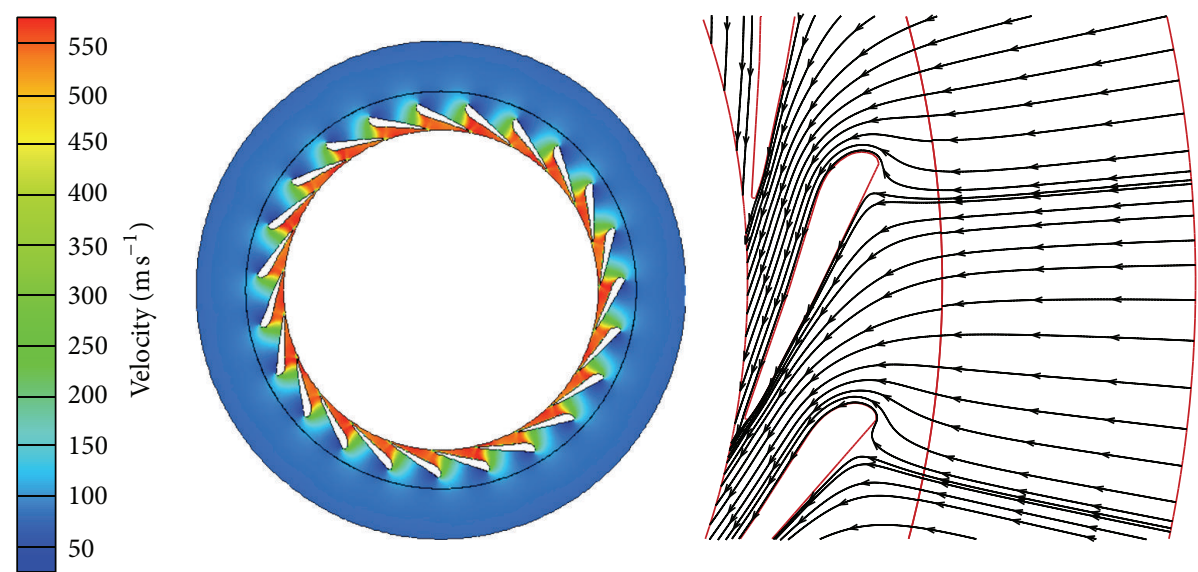

FIGURE 9: Velocity distribution and streamline in the middle section of the stator blade.

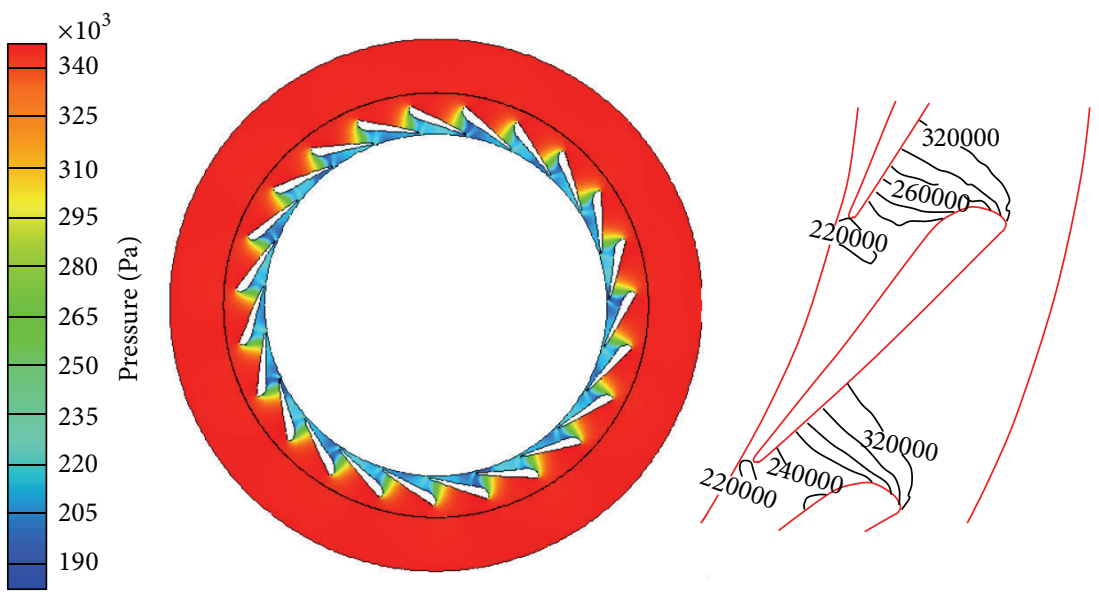

FIGURE 10: Pressure distribution in the middle section of the stator blade.
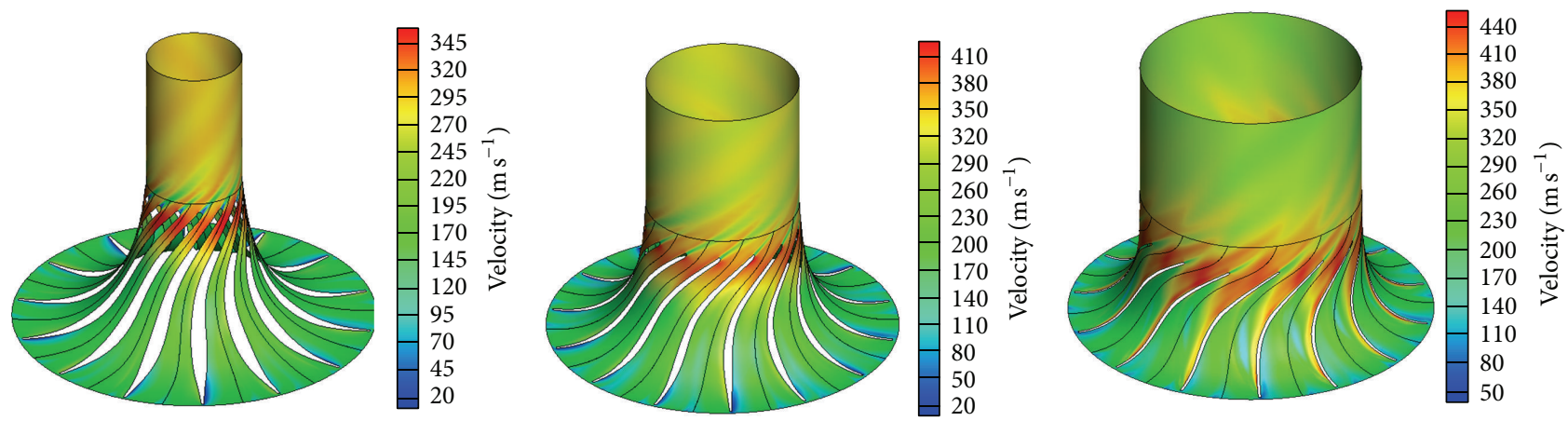

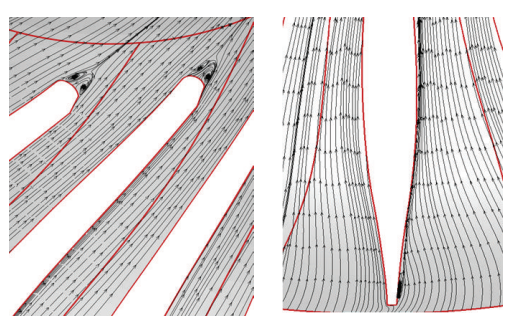

(a)

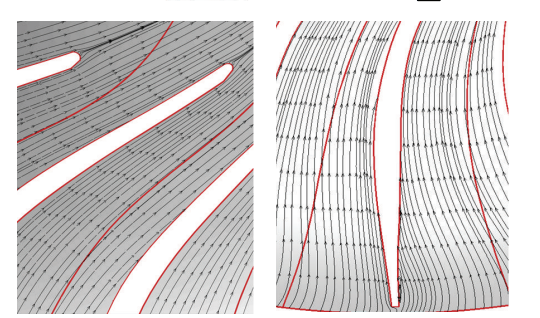

(b)

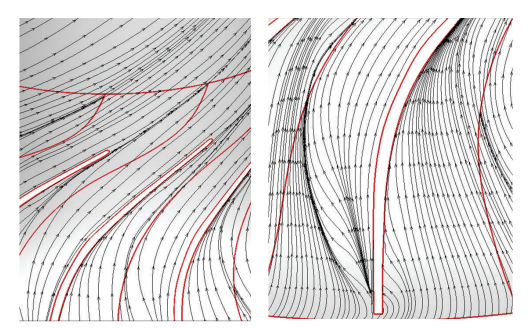

(c)

FIGURE 11: Velocity distribution and local streamline for the sections of (a) 10\%, (b) 50\%, and (c) $90 \%$ blade height. 


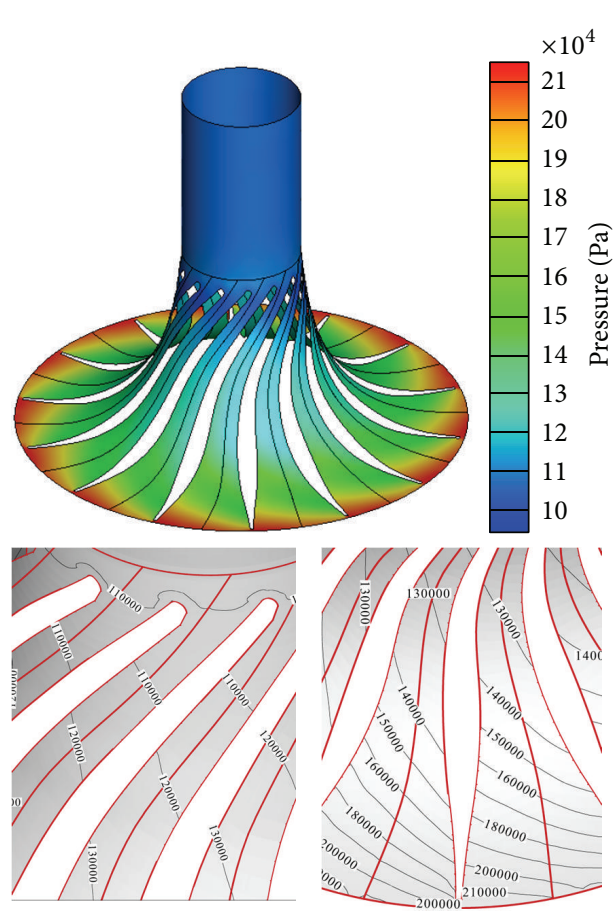

(a)

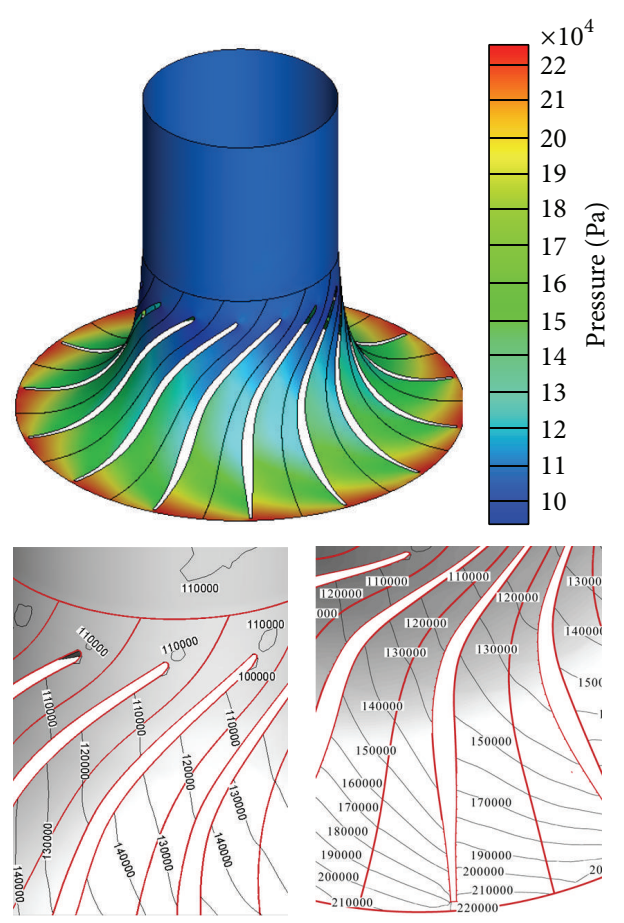

(b)
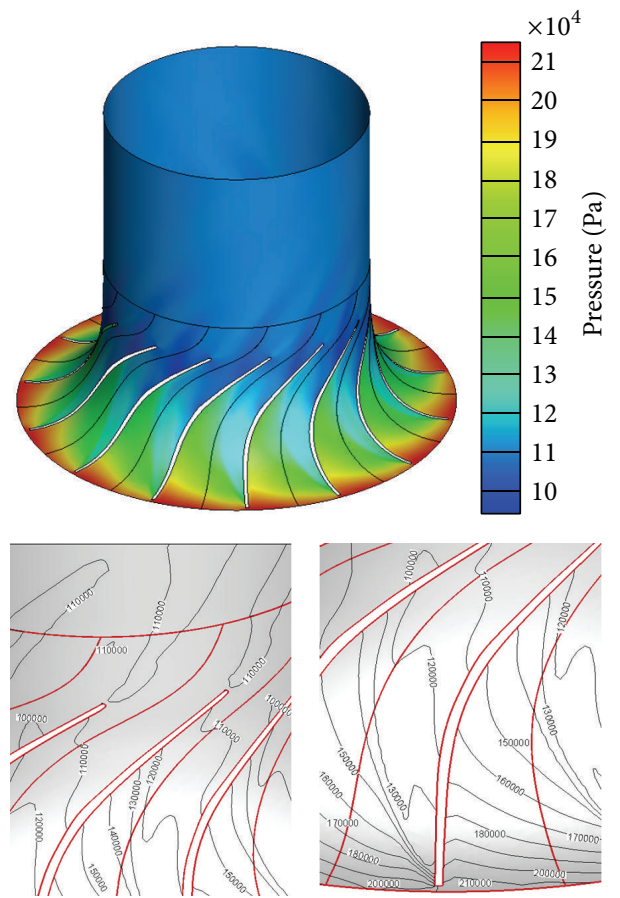

(c)

Figure 12: Pressure distribution for the sections of (a) 10\%, (b) $50 \%$, and (c) $90 \%$ blade height.

3.2. Results and Discussions. Three cases are considered to analyze the effect of different loads on the overall stress of the turbine, as shown in Table 2. For strength analysis, the dimensionless equivalent stress is used, which is the ratio of the equivalent stress to the maximum equivalent stress in the turbine. Figure 16 shows the dimensionless equivalent stress distribution on the rotor blade. The dimensionless thermal stress distribution for Case 1 is shown in Figure 16(a). A maximum thermal stress of $133 \mathrm{MPa}$ occurs at the blade tip of $30 \%$ chord from the leading edge. As observed, a stress concentration region occurs at the blade root of $60 \%$ chord from the leading edge. This is consistent with the high temperature 


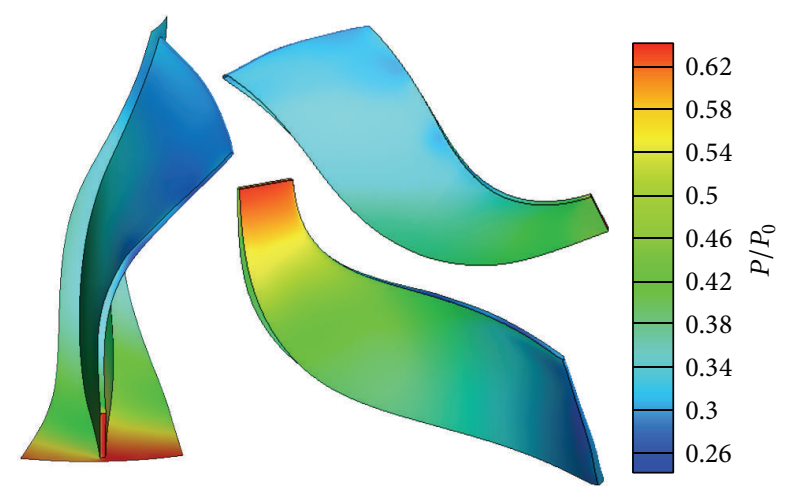

FIGURE 13: Dimensionless pressure distribution on the turbine blade.
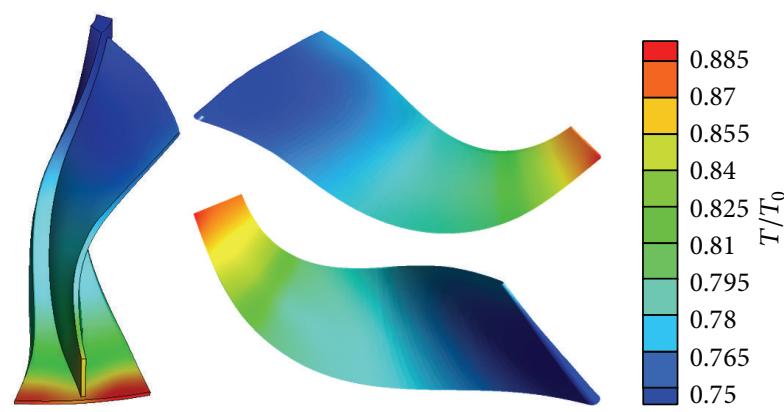

Figure 14: Dimensionless temperature distribution on the turbine blade.

TABLE 2: Different cases considered for stress analysis.

\begin{tabular}{|c|c|c|c|c|}
\hline Cases & $\begin{array}{l}\text { Centrifugal } \\
\text { load }\end{array}$ & $\begin{array}{c}\text { Aerodynamic } \\
\text { load }\end{array}$ & $\begin{array}{c}\text { Thermal } \\
\text { load }\end{array}$ & Results \\
\hline Case 1 & No & No & Yes & $\begin{array}{c}\text { Thermal } \\
\text { stress }\end{array}$ \\
\hline Case 2 & Yes & Yes & No & $\begin{array}{c}\text { Centrifugal } \\
\text { stress }\end{array}$ \\
\hline Case 3 & Yes & Yes & Yes & Total stress \\
\hline
\end{tabular}

gradient in this region obtained in the heat-flow coupling analysis. Figure 16(b) shows the dimensionless equivalent stress distribution for Case 2. A maximum equivalent stress of $347 \mathrm{MPa}$ occurs at the blade root of $40 \%$ chord from the leading edge. Besides, the dimensionless equivalent stress decreases with the increasing blade height. The equivalent stress distribution for Case 3 is shown in Figure 16(c). As observed, the stress distribution is quite similar with that in Case 2, and two stress concentration regions occur on the blade. One is located at the blade tip of $30 \%$ chord from the leading edge, and the other is located at the blade root of $40 \%$ chord from the leading edge. The yield strength of the turbine material is higher than $700 \mathrm{MPa}$, and the maximum equivalent stress is $406 \mathrm{MPa}$, indicating that the micro gas turbine is safe and reliable in operation.
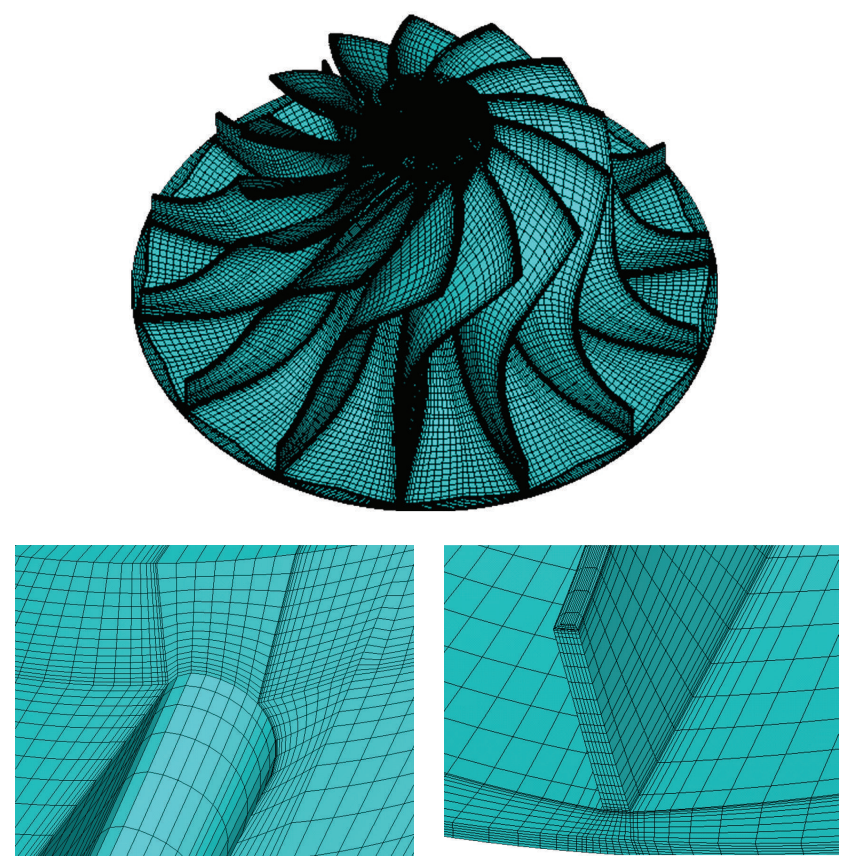

FIGURE 15: Mesh for the rotor blade in the solid domain.

\section{Conclusions}

In this paper, the three-dimensional flow pattern and heat transfer characteristics in a micro gas turbine are studied by using the heat-flow coupling method. The stress distribution of the turbine is also investigated based on the results of the heat-flow coupling analysis. The main conclusions are summarized as follows.

(1) The flow in the stator blade passage is smooth. For the rotor blade passage, a vortex occurs at the leading edge of the pressure surface, and a counter-rotating vortex pair occurs at the trailing edge of the blade. The flow is much smoother at a higher blade height.

(2) In the section of $90 \%$ blade height, the tip clearance has notable effects on the flow, leading to the inhomogeneous flow around the blade tip. The variation in the impeller temperature is relatively smooth, the maximum temperature occurs at the inlet, and the minimum temperature occurs at the blade root around the trailing edge.

(3) A maximum thermal stress of $113 \mathrm{MPa}$ is obtained when only considering the thermal load, indicating that the effects of thermal stress cannot be neglected. When considering the effect of centrifugal load and aerodynamic load, a maximum equivalent stress of $347 \mathrm{MPa}$ is obtained. With a consideration of thermal load, centrifugal load, and aerodynamic load, a maximum equivalent stress of $406 \mathrm{MPa}$ is obtained.

\section{Conflict of Interests}

The authors declare that there is no conflict of interests regarding the publication of this paper. 


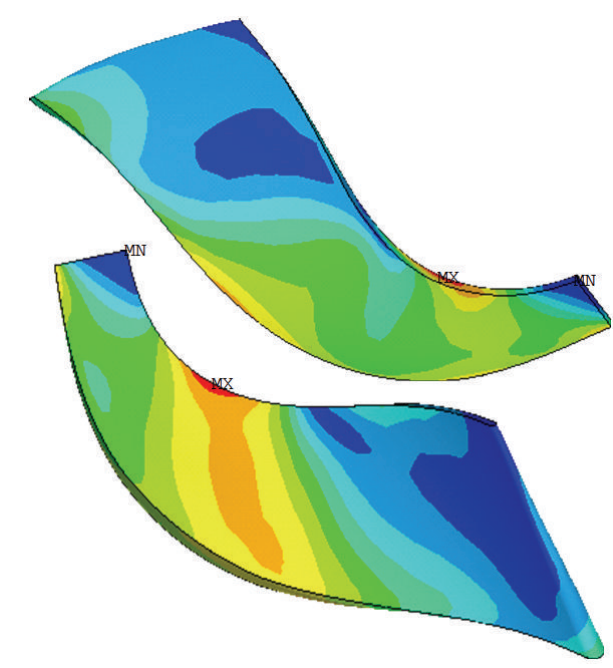

$\begin{array}{llllllllll}0.001 & 0.112 & 0.223 & 0.335 & 0.445 & 0.556 & 0.668 & 0.782 & 0.887 & 1.000\end{array}$

(a) Distribution of dimensionless equivalent stress with thermal load considered

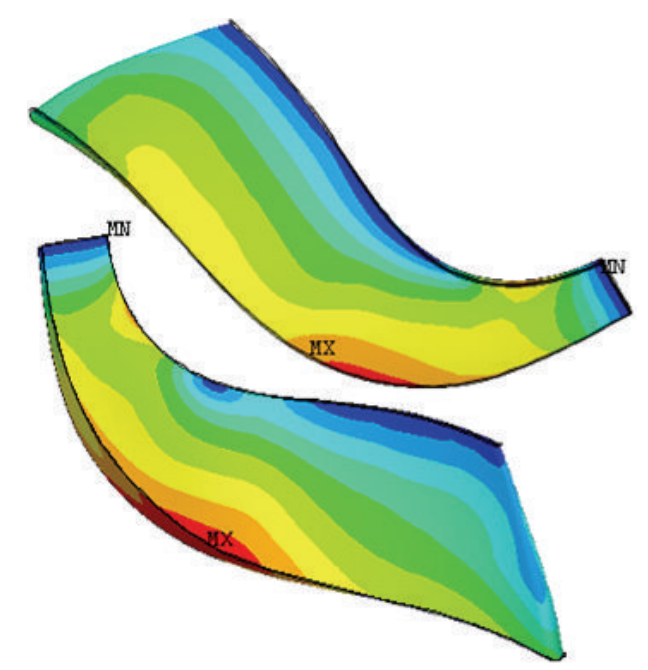

$\begin{array}{llllllllll}0.003 & 0.106 & 0.225 & 0.334 & 0.447 & 0.556 & 0.669 & 0.778 & 0.888 & 1.000\end{array}$

(b) Distribution of dimensionless equivalent stress with centrifugal load and aerodynamic load considered

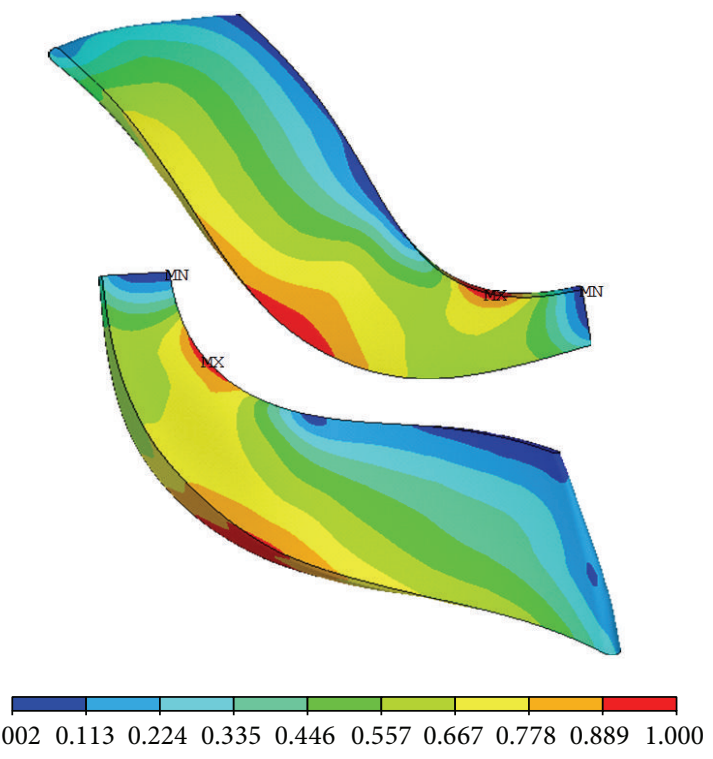

(c) Distribution of dimensionless equivalent stress with centrifugal load, aerodynamic load, and thermal load considered

FIGURE 16: Distribution of dimensionless equivalent stress on the turbine blade.

\section{References}

[1] J. P. Johnston, S. Kang, T. Arima, M. Matsunaga, H. Tsuru, and F. B. Prinz, "Performance of a micro-scale radial-flow compressor impeller made of silicon nitride," in Proceedings of the International Gas Turbine Congress (IGTC '03), Tokyo, Japan, November 2003.

[2] Y. Ribaud, "Internal heat mixing and external heat losses in ultra micro turbine," in Proceedings of the International Gas Turbine Congress (IGTC '03), Tokyo, Japan, November 2003.

[3] V. G. McDonell, R. L. Hack, S. W. Lee, J. L. Mauzey, J. S. Wojciechowski, and G. S. Samuelsen, "Experiences with microturbine generator systems installed in the south coast air quality management district," in Proceedings of ASME Turbo Expo 2003: Power for Land, Sea, and Air, pp. 711-721, Atlanta, Ga, USA, June 2003.

[4] C. Rodgers, "The characteristics of radial turbines for small gas turbines," in Proceedings of ASME Turbo Expo 2003, Power for Land, Sea, and Air, Atlanta, Ga, USA, June 2003.

[5] T. Onishi, S. Bugruburu, O. Derssornes, and Y. Ribaud, "Numerical design and study of a MEMS-based micro turbine," in Proceedings of ASME Turbo Expo 2005: Power for Land, Sea, and Air, Reno-Tahoe, Nev, USA, June 2005.

[6] L. Fu, Y. Shi, Q. Deng, and Z. Feng, "Aerodynamic design and numerical investigation on overall performance of a microradial turbine with millimeter-scale," Journal of 
Engineering for Gas Turbines and Power, vol. 132, no. 3, Article ID 032301, 2010.

[7] D. Bohn and K. Kusterer, "Blowing ration influence on jet mixing flow phenomena at leading edge," in Proceedings of the 37th AIAA Aerospace Sciences Meeting and Exhibit, Reno, Nev, USA, January 1999.

[8] D. Bohn and T. Heuer, "Conjugate flow and heat transfer calculation of a high pressure turbine nozzle guide vane," in Proceedings of the 15th AIAA Computational Fluid Dynamics Conference, Anaheim, Calif, USA, June 2001.

[9] D. Bohn, "Numerical 3-D conjugate flow and heat transfer investigation of a transonic convection-cooled thermal barrier coated turbine guide vane with reduced cooling fluid mass flow," in Proceedings of ASME Turbo Expo 2003: Power for Land, Sea, and Air, Atlanta, Ga, USA, June 2003.

[10] D. Bohn, V. Becker, and K. Kusterer, "3-D conjugate flow and heat transfer calculations of film-cooled turbine guide vane at different operation conditions," ASME Paper 97-GT-23, 1997.

[11] D. Bohn, T. Heuer, and K. Kusterer, "Conjugate flow and heat transfer investigation of a turbo charger: partI: numerical results," in Proceedings of ASME Turbo Expo 2003, Power for Land, Sea, and Air, Atlanta, Ga, USA, June 2003.

[12] J. M. Hill, Y. H. Wu, and B. Wiwatanapataphee, "Analysis of flux flow and the formation of oscillation marks in the continuous caster," Journal of Engineering Mathematics, vol. 36, no. 4, pp. 311-326, 1999.

[13] Y. H. Wu, B. Wiwatanapataphee, and X. Yu, "An enthalpy control volume method for transient mass and heat transport with solidification," International Journal of Computational Fluid Dynamics, vol. 18, no. 7, pp. 577-584, 2004.

[14] S.-Y. Ho and A. Paull, "Coupled thermal, structural and vibrational analysis of a hypersonic engine for flight test," Aerospace Science and Technology, vol. 10, no. 5, pp. 420-426, 2006.

[15] X. Shen, S. Dong, and X. Qi, "The fluid-thermal-structure coupled analysis and optimization of turbine mortise/disc," in Proceedings of the ASME, Gas Turbine India Conference (GTINDIA '12), Mumbai, India, December 2012.

[16] P. V. Krishnakanth, G. N. Raju, R. D. V. Prasad, and R. Saisrinu, "Structural \& thermal analysis of gas turbine blade by using F.E.M.", International Journal of Scientific Research Engineering \& Technology, vol. 2, no. 2, pp. 60-65, 2013.

[17] J. N. Rajadas, A. Chattopadhyay, N. Pagaldipti, and S. Zhang, "Shape optimization of turbine blades with the integration of aerodynamics and heat transfer," Mathematical Problems in Engineering, vol. 4, no. 1, pp. 21-42, 1998.

[18] S. S. Talya, J. N. Rajadas, and A. Chattopadhyay, "Multidisciplinary design optimization of film-cooled gas turbine blades," Mathematical Problems in Engineering, vol. 5, no. 2, pp. 97-119, 1999.

[19] S. S. Talya, A. Chattopadhyay, and J. N. Rajadas, "Multidisciplinary design optimization procedure for improved design of a cooled gas turbine blade," Engineering Optimization, vol. 34, no. 2, pp. 175-194, 2002.

[20] G. Nowak and W. Wroblewski, "Thermo-mechanical optimization of cooled turbine vane," in Proceedings of the ASME Turbo Expo 2007: Power for Land, Sea, and Air, Montreal, Canada, May 2007.

[21] S. Alexandrov, E. Lyamina, and Y.-R. Jeng, "Design of an annular disc subject to thermomechanical loading," Mathematical Problems in Engineering, vol. 2012, Article ID 709178, 9 pages, 2012.
[22] F. R. Menter, "Two-equation eddy-viscosity turbulence models for engineering applications," AIAA journal, vol. 32, no. 8, pp. 1598-1605, 1994.

[23] L. D. Hylton, M. S. Milhec, E. R. Turner, and R. E. York, "Analytical and experimental evaluation of the heat transfer distribution over the surface of turbine vanes," Tech. Rep. NASA-CR-168015, 1983.

[24] E. R. Turner, M. D. Wilson, L. D. Hylton, and R. M. Kaufman, Turbine Vane External Heat Transfer, vol. 1 of Analytical and Experimental Evaluation of Surface Heat Transfer Distributions with Leading Edge Showerhead Film Cooling, NASA Lewis Research Center, Cleveland, Ohio, USA, 1985.

[25] R. Dambach, H. P. Hodson, and I. Huntsman, "An experimental study of tip clearance flow in a radial inflow turbine," Journal of Turbomachinery, vol. 121, no. 4, pp. 644-650, 1999. 


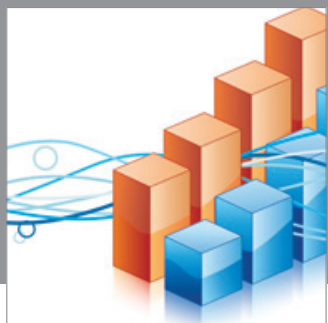

Advances in

Operations Research

mansans

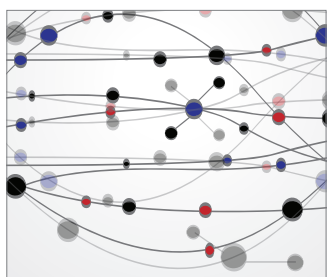

The Scientific World Journal
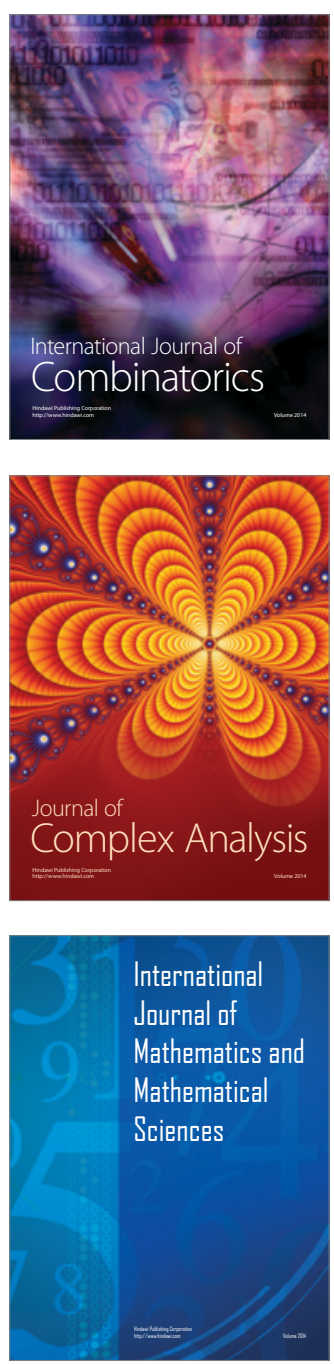
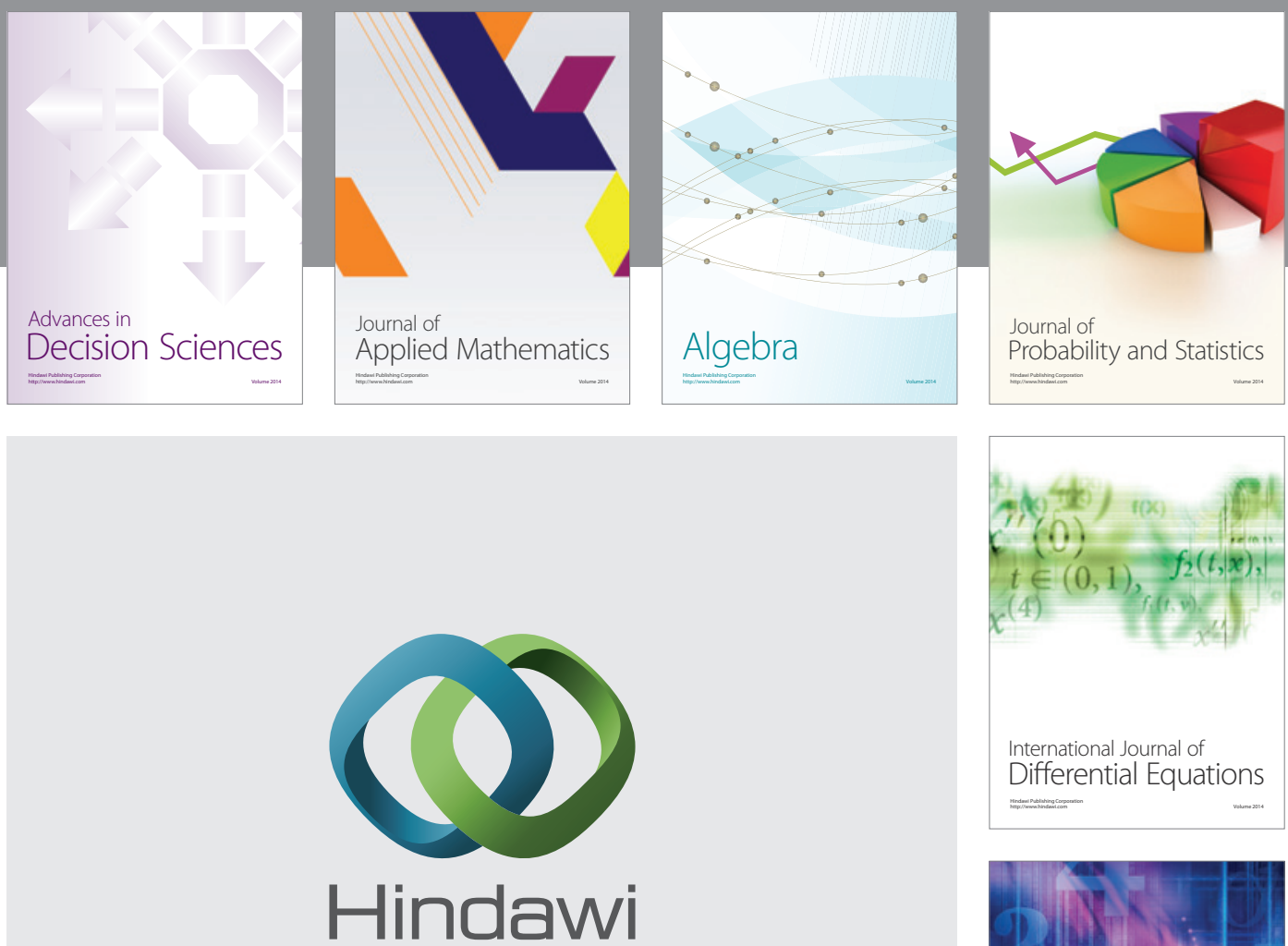

Submit your manuscripts at http://www.hindawi.com
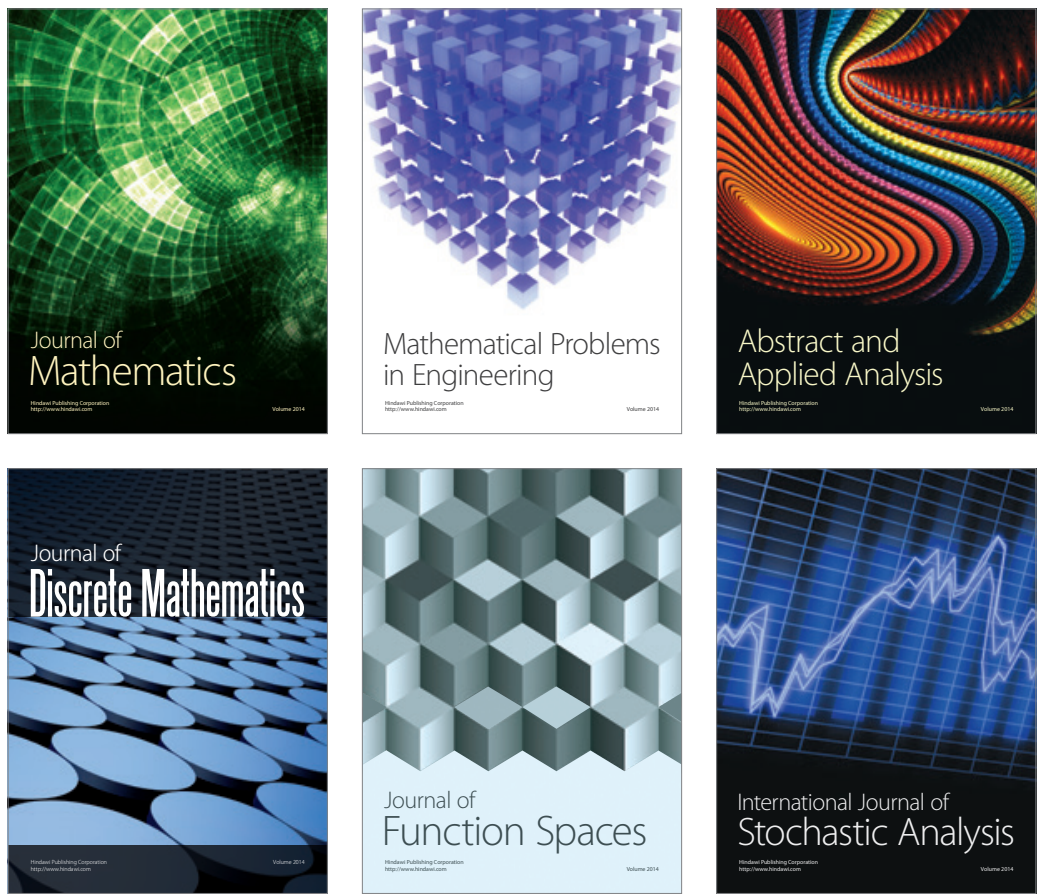

Journal of

Function Spaces

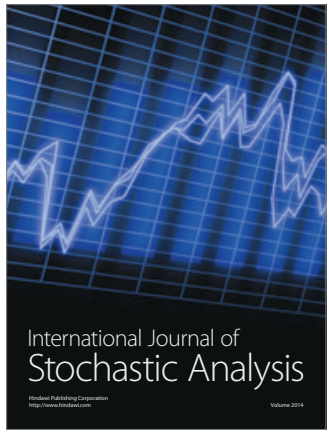

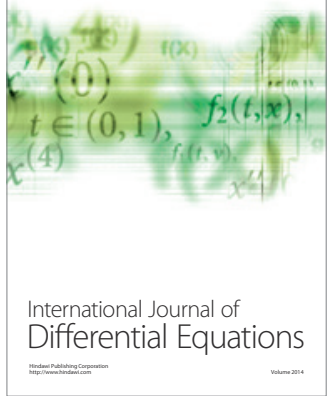
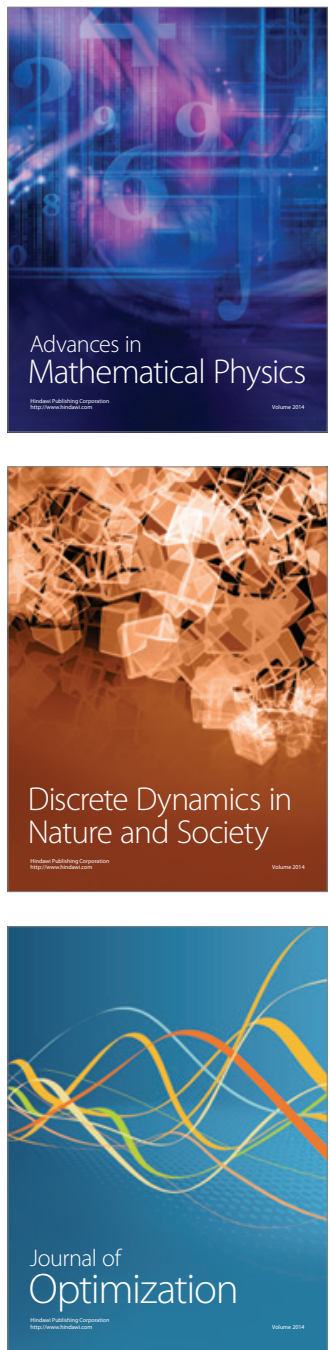